\title{
The measurement and interpretation process to determine the state of stress in rock including the effects of fluid pressure
}

\author{
I Gray Sigra Pty Ltd, Australia
}

This abstract examines the fundamentals of stress in the ground, from the basic active and passive pressure limitations on the state of stress in a soil to focus on the state of stress in rock. In the case of sedimentary rocks, the influence of soil stress may still be visible; however, the more important effects in most rocks are likely to be those derived from the subsequent loading of the rock mass. This loading is derived from gravitational effects and the strains imposed on the rock mass externally. These strains are derived from true tectonic plate effects frequently heavily influenced, or indeed totally masked, by local features such as faulting and folding. The other source of loading is gravitational and, in this case, the stress derived from this is very dependent on Poisson's ratio: an important number that is often extremely difficult to determine in many sedimentary rocks. Thermal loading and, in particular, cooling are also important in determining the state of stress in rock. This is especially the case in igneous bodies.

In addition, the concepts of effective stress within the ground are examined. The premise of effective stress, as applied to soil mechanics, is quite inappropriate when applied to rock that lacks the point contact concept that is fundamental to soils. Fracture area and fracture coalescence become very important in determining the state of effective stress in a fractured rock mass. Indeed, the concept of effective stress in a rock mass cannot be applied to a plane but rather to a surface of interest, usually a potential failure surface.

One interesting theoretical finding of this study is that under a state of zero lateral strain, a rock mass may have no effective lateral stress unless it undergoes failure, which leads to lateral loading.

The state of effective stress in porous media is vital to the understanding of permeability change. The concept of stress path that is familiar to the soil mechanics practitioner gains a new dimension when applied to stress sensitive unconventional petroleum reservoirs such as coals or shales.

Finally, the paper turns to what can be measured and how you might measure it. It examines the practise and concepts of overcoring, hydrofracturing and examination of failure, be it borehole breakout or on fault planes in determining the state of stress in the ground. In many cases, the most important component of stress is that imposed by the fluid, be it water, gas or petroleum liquid, provided that the material has internal surfaces on which that pressure may act. The measurement of fluid pressure in the ground is therefore vital to an understanding of its behaviour. 
including the effects of fluid pressure 